\title{
INFLUENCIA DE LA ENSENANANZA ASISTIDA POR ORDENADOR EN EL RENDIMIENTO Y LAS IDEAS DE LOS ALUMNOS EN ELECTRICIDAD
}

\author{
GÓMEZ CRESPO, M.A. \\ IB Victoria Kent. C/ La Plata s/n. 28850 Torrejón de Ardoz. Madrid.
}

\begin{abstract}
SUMMARY
This paper presents an experience about the use of computers in learning electricity. It was carried out with students of $3^{\circ}$ BUP. It analyses the efficacy of using a certain computer programme versus the traditional work in the classroom.
\end{abstract}

Durante los últimos años la informática está empezando a ocupar un puesto importante en la enseñanza y en el sistema educativo. Los ordenadores van poco a poco introduciéndose en las aulas y los laboratorios escolares, bien utilizando herramientas de trabajo generales -hojas de cálculo, bases de datos, etc.- o bien utilizando herra* mientas más específicas -software educativo-especialmente diseñadas para la enseñanza.

Las ventajas que tradicionalmente se atribuyen a la enseñanza asistida por ordenador (EAO) frente a la enseñanza tradicional son varias. Entre otras, pueden citarse: la motivación que produce en los estudiantes; la personalización del proceso de aprendizaje, permitiendo que cada sujeto aprenda a su propio ritmo; y la informacion inmediata que proporciona al alumno sobre sus respuestas, permitiéndole volver sobre sus pasos. A su vez, Ia EAO, frente a la enseñanza tradicional, facilita la tarea del profesor a la hora de utilizar diferentes estrategias didácticas con distintos grupos de alumnos. Todo ello hace que la EAO sea un instrumento atractivo tanto para alumnos como para profesores. Pero no siempre es así y, aunque atractivo, en bastantes ocasiones el software educativo queda en un mero pretexto para la utilizacion del ordenador. No debemos olvidar que, al hablar de enseñanza asistida por ordenador, la palabra clave es enseñanza y no ordenador (Vacas y Juanes 1991). Por ello, la utilización del ordenador en el aula no debe ser un fin, sino un medio para alcanzar determinados objetivos psicopedagógicos (Rosa 1985). En consecuencia, a la hora de elegir un determinado software educativo debemos plantearnos algunas preguntas: $i$ sirve para aicanzar los objetivos previstos?, ¿qué aporta al proceso de enseñanza.aprendizaje?, ¿mejora el rendimiento de los alumnos, su motivación?, etc. En definitiva, debe buscarse un software educativo, debidamente evaluado, que además de entretener y motivar sea un instrumento eficaz en el proceso de aprendizaje de los alumnos.

Hasta ahora, en España se han utilizado una cierta cantidad de programas de EAO, pero son pocas las experiencias en las que se evalúe dicho material. Los resultados de dichas experiencias llevan a Ia idea de que la utilización de la EAO no siempre produce una mayor eficacia en el proceso de aprendizaje. Así, por ejemplo, Bautista (1987), trabajando con alumnos de EGB en el estudio de la geometría a través de LOGO, y Martín y otros (1988), utilizando programas de simulación en física con alumnos universitarios, no encuentran diferencias significativas entre los que trabajan con ordenador y los que no. En el otro extremo, tanto Vidal y otros (1985), trabajando con disoluciones, como Del Bario y 
Morales (1989), en el estudio de la mecánica (ambos con alumnos de $2^{\circ}$ de BUP), encuentran diferencias a favor de los grupos que trabajan con ordenador. Con respecto a la motivación, prácticamente todos los autores están de acuerdo en el efecto favorable del uso de este instrumento.

Los resultados anteriormente citados, aunque en cierto modo contradictorios desde el punto de vista de la utilización de la EAO, puede que no lo sean tanto si tenemos en cuenta que en cada caso se ha utilizado un software diferente, con grupos de distinto nivel, en distintas condiciones de aplicación y persiguiendo objetivos diferentes. Todo ello lleva a la idea de que es muy necesario evaluar el software que se utiliza, pero hay que hacerlo en las condiciones y con los instrumentos de análisis adecuados a cađa caso.

Este trabajo presenta una experiencia en la que se analiza la eficacia de un determinado programa de EAO frente aI trabajo tradicional en el aula. La experiencia se ha diseñado en torno a la utilización del módulo «Resistencia Eléctrica” del paquete MICROLAB (Degem Systems) descrito y utifizađo por otros autores (por ejemplo, López y Martínez del Amor 1989, Herrero 1990). Se investiga la incidencia que tiene la utilización de este material en el estudio de la corriente y los circuitos eléctricos, la evolución de las concepciones de los alumnos sobre dichos contenidos y el rendimiento en la resolución de este tipo de problemas.

\section{CONCEPCIONES DE LOS ALUMNOS SO- BRE LA CORRIENTE Y LOS CIRCUITOS ELECTRICOS}

Son muchos los autores que sostienen que la enseñanza de la ciencia debe partir de las ideas que los alumnos poseen para poder modificarlas. Por ello, en los últimos tiempos, gran parte de la investigación sobre didáctica de las ciencias ha estado dedicada a estudiar los conocimientos previos y concepciones espontáneas de los alumnos sobre las diversas materias que conforman el currículo.

Estos conocimientos previos o concepciones espontáneas de los alumnos, descritos en numerosas publicaciones (por ejemplo, Driver et al. 1985, Hierrezuelo y Montero 1988), difieren de unas áreas a otras del currículo, no sólo en sus contenidos, sino también en su naturaleza. Sin embargo, generalizando, pueden encontrarse algunas características comunes entre todos ellos (Pozoet al. 1991 a y $1991 \mathrm{~b}$ ), que a grandes rasgos pueden describirse como sigue: son construcciones personales de los alumnos elaboradas de forma espontánea en su interacción cotidiana con el mundo que les rodea; son incoherentes desde el punto de vista cientifico, aunque no tienen porque serlo desde el punto de vista deI alumno; resultan bastante estables y resistentes al cambio; persisten a pesar de la instrucción científica; tienen un carácter implícito frente al carácter explícito de las ideas científicas; buscan la utilidad más que la verdad.
En el campo de la corriente y de los circuitos eléctricos, del que se ocupa este trabajo, existe una amplia bibliografía sobre las ideas de los alumnos de diferentes niveles de enseñanza y de distintos países. De forma breve, estas ideas pueden resumirse de la siguiente manera:

- No hace falta cerrar un circuito para que haya corriente eléctrica (modelo unipolar). Para hacer lucir una bombiIla con una pila basta un solo cable o, en el caso de que haya dos, no hay corriente en el camino de regreso (Shipstone 1985 y 1988 , Osborne y Freyberg 1985).

- La corriente no completa un circuito. Aparecen dificultades a la hora de fijar el sentido de la circulación de la corriente; por ejemplo, para algunos alumnos, la corriente sale por los dos polos y se gasta en los elementos del circuito (Shipstone 1985, Osborne y Freyberg 1985).

- La corriente eléctrica se gasta a lo largo del circuito; la intensidad va disminuyendo según pasa por los distintos elementos del circuito (Shipstone 1985).

- La diferencia de potencial se interpreta como una consecuencia del flujo de corriente y no como la causa de éste (Cohen et al. 1983, Shipstone 1984).

- Los generadores representan un almacén de electricidad. Las pilas son vistas como almacenes de corriente eléctrica que se va gastando en los distintos elementos del circuito (Shipstone 1985).

\section{TRABAJO EMPÍRICO}

\section{Objetivos}

Dentro del marco específico de los contenidos de electricidad de $3^{\circ}$ de BUP y para un determinado módulo de EAO, se pretende estudiar:

1. Las diferencias que la aplicación de la EAO provoca, frente al trabajo tradicional en el aula, en el número de intentos que el alumno necesita para resolver un problema, ser consciente de sus propios errores y llegar a una solución correcta.

2. El efecto que sobre las «ideas previas» $\mathrm{y}$ «concepciones erróneas» đe los alumnos tiene el uso de este módulo.

3. Cómo afecta, al rendimiento de los alumnos en la resolución de problemas de electricidad, la utilización de este módulo de EAO.

\section{Sujetos}

La experiencia se realizó con 60 alumnos de $3^{\circ}$ de BUP que cursaban la asignatura Física y Química. El curso anterior no habían estudiado electricidad y previamente al experimento todos habían trabajado con otros módu- 
los de EAO. Los alumnos fueron distribuidos de la siguiente manera:

Grupo experimental: 30 alumnos elegidos al azar (repartidos en dos subgrupos de 15 alumnos cada uno).

Grupo de control: 30 alumnos elegidos al azar (repartidos en dos subgrupos de 15 alumnos cada uno).

\section{Materiales utilizados y diseño de la experiencia}

Con el grupo experimental se utilizó un conjunto de 8 problemas de electricidad del módulo «Resistencia Eléctrica» (MICROLAB, Degem Systems). En ellos se plantean al alumno diversas situaciones en las que tiene que aplicar los conceptos de resistencia eléctrica, intensidad y diferencia de potencial a la resolución de circuitos eléctricos. Los alumnos podían acceder a toda la información que el programa proporciona, solicitando aquellos datos que les hacían falta en cada momento.

Con el grupo de control se utilizaron los mismos 8 problemas, secuenciados de la misma manera, transcritos a papel con todos los datos necesarios para su resolución. Además se les facilitó una hoja con toda la información extra que el ordenador proporcionaba al grupo experimental.

En ambos casos, el trabajo se desarrolló en grupos de 3 alumnos en dos sesiones de 45 minutos cada una. En el grupo experimental, el ordenador suministraba toda la información que los alumnos necesitaban e informaba si el resultado propuesto por ellos era correcto o no. En caso de que fuera correcto, los sujetos pasaban al problema siguiente y, en caso de que fuera incorrecto, les indicaba que volvieran a intentarlo. En el grupo de control, el profesor suministró a cada grupo de alumnos un problema escrito y una hoja con la misma información que el ordenador proporcionaba al grupo experimental. Estos alumnos proponían una solución y el profesor les indicaba si era correcta o no. En caso afirmativo pasaban af problema siguiente $y$, en caso negativo, volvían a intentarlo hasta que obtuvieran una solución correcta.

La experiencia se llevó a cabo durante las dos semanas siguientes a la finalización de la unidad didáctica «Electricidad», una vez que se había realizado el proceso de evaluación de dichos contenidos. Para ello se utilizaron horas de «desdoble», lo que exigía la participación de dos profesores - uno realizaba la experiencia en su aula habitual (grupo de control) y el otro realizaba la experiencia con los ordenadores (grupo experimental)-. Con el fin de evitar influencias de la variable profesor, se alternaron los profesores, de manera que en cada grupo (experimental y de control) de 30 alumnos, la mitad hubiera trabajado con un profesor y la otra mitad con el otro.

\section{Instrumentos de evaluación}

La evaluación de la experiencia se llevó a cabo mediante tres instrumentos: a) Número de intentos necesarios para la resolución de cada problema. Se recogió la información proporcionada por la utilidad «Evaluación» del propio programa de EAO.

b) Concepciones de Ios alumnos. Se utilizó un cuestionario con ítems de respuesta cerrada sobre ideas previas de electricidad. Se aplicó a mitad de curso (pretest) cuando los alumnos no habían estudiado todavía nada de dicho tema. Posteriormente, una vez finalizada la experiencia ( 4 meses después del pretest), se aplicó el mismo cuestionario (postest).

c) Rendimiento de los alumnos. Se utilizó una prueba de evaluación sobre los contenidos de electricidad (integrada en las evaluaciones normales que se llevan a cabo durante el curso). Esta prueba se aplicó después de terminar la unidad didáctica «Electricidad», una semana antes de comenzar la experiencia (pretest). En esta prueba (de carácter abierto) se plantean cuestiones y ejercicios típicos de electricidad, en los que el alumno debe aplicar los conceptos estudiados a la resolución de circuitos eléctricos. Posteriormente, dos semanas después de finalizar la experiencia se aplicó otra prueba de evaluación (postest) similar a la utilizada como pre-test y con ejercicios de distinto tipo a los utilizados en el tratamiento experimental.

\section{Análisis de datos}

Se realizaron los siguientes análisis:

- Número de intentos necesarios para resolver los problemas.

Las diferencias entre las medias de intentos realizados por cada grupo, se analizaron mediante una prueba $t$.

- Influencia del tratamiento sobre las ideas previas.

Aunque los dos grupos, experimental y de control, se formaron al azar, debido al número de sujetos relativamente pequeño que los componen, era previsible que existieran diferencias iniciales en variables relevantes. Por ello se utilizó en la investigación un diseño experimental basađo en un pretest y un postest, aplicándose un mismo cuestionario de ideas previas, antes y después de tratamiento, manteniendo una diferencia de cuatro meses entre una y otra aplicación.

A los resultados obtenidos, se les aplicó un análisis de covarianza (ANACOVA), en el cual la variable dependiente era el número de respuestas correctas en el cuestionario de ideas previas y la variable independiente, el grupo; siendo la covariante el número de respuestas correctas de cada grupo en el pretest.

- Influencia del tratamiento sobre el rendimiento de los alumnos.

Al igual que se manifestó en el análisis anterior, aunque los sujetos se agruparon al azar y no tenían instrucción previa en electricidad, era previsible que existieran dife- 
rencias iniciales entre los grupos que afectaran a la variable dependiente. Por ello se utilizó en la investigación un diseño experimental basado en un pretest y un postest (véase más arriba la descripción de los instrumentos utilizados).

A los resultados obtenidos se les aplicó un análisis de covarianza (ANACOVA) en el que la variable dependiente era la puntuación obtenida en el postest y la variable independiente, el grupo; siendo la covariante la puntuación obtenida en el pretest.

\section{RESULTADOS}

- Número de intentos necesarios para resolver los problemas.

El análisis realizado muestra que existen diferencias significativas entre los grupos experimental y de control $(t=3,37 p<0,005)$. Se observa que los alumnos que utilizan el ordenador necesitan más intentos para resolver los ocho problemas que los alumnos que los resuelven por el método tradicional de lápiz y papel.

En Ia tabla I se muestran el número medio de intentos que han necesitado los alumnos para resolver los 8 problemas de que consta la experiencia.

\section{Tabla I}

Media de intentos que han necesitado los alumnos de cada grupo para resolver los 8 problemas.

\begin{tabular}{|l|c|c|}
\hline & Grupo experimental & Grupo de control \\
\hline Media de intentos & 16,6 & 11,3 \\
\hline Desviación típica & 4,6 & 0,9 \\
\hline
\end{tabular}

Se observa que el grupo experimental (ordenador) realiza más intentos para poder resolver el mismo número de problemas que el grupo de control (lápiz y papel), aunque en el mismo tiempo. Además, puede verse que la desviación es mayor en el grupo experimental, lo que indica una mayor dispersion entre el número de intentos que ha necesitado cada alumno frente a los que han necesitado los alumnos del grupo de control.

Estos resultados podrían mostrar que los alumnos deI grupo que trabaja con el ordenador prueban más sus posibles soluciones, sin miedo a que el resultado sea erróneo. Frente a esto, los alumnos que han trabajado en el aula y tienen que consultar sus soluciones con el profesor, buscan una mayor seguridad antes de someterlas a contraste.

De forma cualitativa, se ha analizado también el número medio de intentos que cada grupo ha necesitado por cada problema (Fig. 1).
Figura I

Media de intentos por grupo que ha sido necesaria para resolver cada problema.

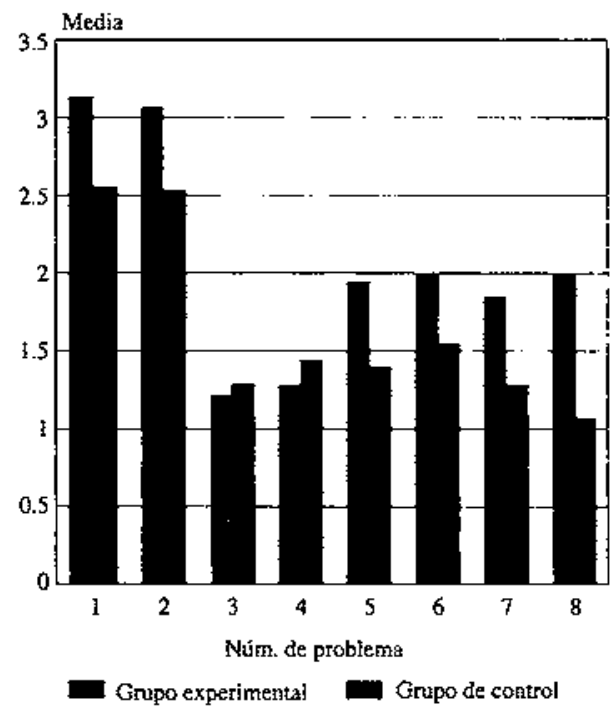

En la figura vemos que, en general, como ya se ha descrito más arriba, el grupo que trabaja con el ordenador realiza más intentos para resolver los problemas que el grupo que trabaja con lápiz y papel. Sin embargo, se observa que hay dos problemas en los que, aunque las diferencias entre grupos son muy pequeñas, la situación se invierte.

Se puede ver también que los dos primeros problemas son los que más número de intentos necesitan para ambos grupos, alrededor de tres intentos, disminuyendo para el resto de los problemas. Esto parece lógico si se tiene en cuenta que, aunque los alumnos ya estaban familiarizados con otros módulos de EAO y con los problemas de electricidad, necesitaron un cierto tiempo para familiarizarse con el tipo de problema y su presentación.

- Influencia del tratamiento sobre las ideas previas.

El análisis de covarianza (ANACOVA) muestra que no existen diferencias significativas (para un nivel de significación $p=0,05$ ) entre Ios grupos experimental y de control debidas al tratamiento recibido. En la tabla II se muestran las medias obtenidas por cada grupo en el pretest y postest.

\section{Tabla II}

Medias obtenidas por cada grupo en el cuestionario de ideas previas (puntuación máxima: 1).

\begin{tabular}{|l|c|c|}
\hline & Gupo experimental & Grupo de control \\
\hline Media pretest & 0,55 & 0,46 \\
\hline Media postest & 0,83 & 0,75 \\
\hline
\end{tabular}


Se observa que, a lo largo del curso, los dos grupos han mejorado su puntuación media debido a la instrucción recibida, aunque las diferencias existentes entre ambos no pueden ser atribuidas al tratamiento experimental.

- Influencia del tratamiento sobre el rendimiento de los alumnos en electricidad.

El análisis de covarianza (ANACOVA) muestra que existen diferencias significativas $(F=6,3 p<0,05)$ en el rendimiento general de los alumnos en electricidad debidas al tratamiento experimental. Las puntuaciones medias obtenidas por cada grupo se muestran en la tabla III.

Tabia III

Puntuaciones medias obtenidas por cada grupo para el rendimiento en electricidad (puntuación máxima: 1).

\begin{tabular}{|l|c|c|}
\hline & Grupo experimental & Grupo de control \\
\hline Media pretest & 0,50 & 0,44 \\
\hline Media postest & 0,67 & 0,47 \\
\hline
\end{tabular}

Se observa que, aunque ambos grupos mejoran su puntuación al pasar del pretest al postest, el grupo que ha trabajado el módulo de EAO obtiene un aumento en su puntuación media sensiblemente superior al grupo que ha trabajado con lápiz y papel. Parece que el añadir ocho problemas más tiene una influencia mínima en el rendimiento de los alumnos que han trabajado de forma tradicional. Sin embargo, a los alumnos que trabajan con el ordenador, les supone un refuerzo suplementario sobre lo aprendido anteriormente.

\section{CONCLUSIONES}

Como una aportación más a lo que ya se ha discutido en los últimos años sobre la enseñanza asistida por ordenador y su influencia en el grado de motivación de los alumnos, esta investigacion presenta un estudio comparativo entre este tipo de enseñanza y la enseñanza tradicional.

En los resultados obtenidos puede verse como la EAO hace que el número de intentos que los alumnos utilizan para solucionar un problema sea más alto que cuando tienen que consultar al profesor. Esto puede interpretar- se como un síntoma de la desinhibición del alumno frente a la máquina, que le hace aumentar el número de ensayos, "prueba para ver qué pasa" hasta que da con la solución correcta. La actitud del alumno que trabaja con el profesor piensa más su respuesta antes de someterla a juicio. Sin embargo, si tenemos en cuenta que los alumnos que han trabajado con EAO obtienen un mejor rendimiento general en electricidad, parece que el mayor número de intentos -ya sean correctos o incorrectospermite que, por el método de ensayo-error, el alumno vaya haciéndose consciente de sus errores hasta que llega a la respuesta correcta, adquiriendo más seguridad en el dominio de los conceptos y de las estrategias de resolución de problemas.

Por otra parte, puede verse que la utilización de la EAO no provoca ninguna diferencia en las concepciones de los alumnos sobre la electricidad, resultado que en principio se esperaba, puesto que el módulo experimental que se ha utilizado estaba fundamentalmente encaminado a trabajar sobre la resolución de problemas. Sin embargo, el tratamiento experimental sí ha demostrado ser eficaz a la hora de mejorar el rendimiento de Ios alumnos en electricidad. Este hecho es importante y resultaría bastante interesante estudiar si estos resultados, obtenidos para unos contenidos concretos y con unos módulos concretos de EAO, son generalizables a otros contenidos del currículo y con otros módulos diferentes de EAO.

En último lugar, con referencia a uno de los aspectos en que más hincapié se hace al hablar de la introducción del ordenador en el aula -la motivacion-, en general se observa que los alumnos muestran una buena disposición hacia este tipo de enseñanza. Pero, también es cierto que, después de haber trabajado con varios módulos de $E A O$, un grupo significativo de alumnos manifiesta sus preferencias por la enseñanza tradicional. Esto está de acuerdo con las investigaciones que se han llevado a cabo sobre los distintos estilos de motivación de los alumnos y la necesiđad de utilizar distintas estrategias didácticas en función de los distintos modelos de motivación (Martín-Díaz y Kempa 1991, Bacas y MartínDíaz 1992).

\section{AGRADECIMIENTOS}

Debo agradecer aquí la colaboración inestimable de Carmen Marín que ha hecho posible llevar a buen fin el trabajo con los alumnos en el aula. A su vez, debo expresar mi agradecimiento a J. I. Pozo por sus acertados comentarios y stgerencias. 


\section{REFERENCIAS BIBLIOGRÁFICAS}

BACAS,P.y MARTÉN-DÍAZ, M.J., 1992. Distintasmotivaciones para aprender ciencias. (Ed. Narcea SA/MEC).

BAUTTSTA, A, 1987. Estudio piloto sobre el efecto del ordenador en la adquisición de conceptos matemáticos en alumnos de $5^{\circ}$ y $7^{\circ}$ de EGB, Enseñanza de las Ciencias, 5(3), pp. 205-210.

COHEN, R., EYLON, B. y GANIEL, U., 1983. Potential difference and current in simple electric circuits: A study of student's concepts, American Journal of Physics, 51(5), pp. $407-412$

DEL BARRIO,F.J. y MORALES GARCIA, J.A., 1989. ¿Pueden ayudar las simulaciones con ordenador a provocar en los alumnos un cambio en sus jdeas sobre mecánica?Actas del III Congresolnternacional sobre la Didáctica de las Ciencias $y$ de las Matemáticas.

DRIVER, R., GUESNE, E. y TIBERGHIEN, A., 1985. Children's ideas in science. Milton Keynes: Open University Press. (Trad., 1989, Ideas cientificas en la infancia yla adolescencia, Morata-MEC).

HERRERO, F., 1990., Microlab para enseñar Física, Apuntes de Educación, 37 , pp. 12-15.

HIERREZUELO, J. y MONTERO, A., 1988. La ciencia de los alumnos. (Laia-MEC).

LÓPEZ, F. y MARTÍNEZ DEL AMOR, P., 1989. Experiencia sobre resolución de problemas de física con ordenador. Taller y exposición de la investigación realizada con alumnos de bachillerato. Actas del HI Congreso Internacional sobre la Didáctica de las Ciencias y las Matemáticas.

MARTIN, E., ZAMARRO, J.M. y MENÉNDEZBARZANALLANA, R, 1988. Losordenadoresenla enseñanza de la física: la gravitación universal, Revista Española de Física, 2(1), pp. 46-50.
MARTÍN DÍAZ, M.J. y KEMPA, R.F., 1991. Los alumnos prefteren diferentes estrategias didácticas de la enseñanza de las ciencias en función de sus características motivacionales, Enseñanza de las Ciencias, 9(1), pp. 59-68.

OSBORNE, R. y FREYBERG, P., 1985. Learning in science. The implications of children'science. (Heinemann). (Trad., 1991, El aprendizaje de las ciencias. Implicaciones de la ciencia de los alumnos, Narcea).

POZO, J.1., LIMÓN, M., SANZ, A. y GÓMEZ CRESPO, M.A., I99 la. Conocimientos previos y aprendizajeescolar, Cuadernos de Pedagogía, 188, pp. 12-14.

POZO, J.I., SANZ, A., GÓMEZCRESPO, M.A. y LIMÓN, M. 1991b. Las ideas de los alumnos sobre la ciencia: una interpretación desde la psicología cognitiva, Enseñanza de las Ciencias, 9(1), pp. 83-94.

ROSA, A., 1985. La fillosofía del uso del ordenador en el ámbito educativo, en Pfeifer, A. y Galván, J., Informática y escuela.

SHIPSTONE, D.M., 1984. A study of children's understanding of electricity in simple D.C. circuits, European Journal of Science Education, 6(2), pp. 185-198.

SHIPSTONE, D., 1985. Electricity in simple circuits, en Driver, R., Guesne, A. y Tiberghien, A., Children's ideas in science. (Open University Press). (Trad, 1989, Ideas cientificas en la infancia y la adolescencia, Morata-MEC).

SHIPSTONE, D., 1988. Pupils understanding of simple electrical circuits, some implication for instruction, Physics Education, 23, pp. 92-96.

VACAS, J.M. y JUANES, J.A., 1991. Creación de un programa docente informatizado, Apuntes de Educación, (42), pp. $2-5$.

VIDAL, J.A., ROMERO, F. y REQUENA, A., 1985. Enseñanza basada en ordenador: una experiencia en BUP, Enseñanza de las Ciencias, 3(2), pp. 100-107. 